\title{
Cross-Layer optimization of the packet loss rate in mobile videoconferencing applications
}

\author{
R. Rivera-Rodríguez ${ }^{* 1,2}$, O. E. Olivares-Domínguez ${ }^{2}, A$. Serrano-Santoyo ${ }^{3}$ \\ 1, Universidad Autónoma de Baja California \\ Facultad de Ingeniería, Campus Mexicali \\ Obregon y Julián Carrillo s/n C.P. 21100 Mexicali, Baja California Norte, México \\ *rrivera@cicese.mx \\ ${ }^{2}$ Centro de Investigación Científica y de Educación Superior de Ensenada CICESE \\ División de Física Aplicada, Departamento de Electrónica y Telecomunicaciones \\ Carretera Ensenada-Tijuana 3918 Zona Playitas C. P. 22860 Ensenada, Baja California, México \\ ${ }^{3}$ Centro de Investigación Científica y de Educación Superior de Ensenada CICESE \\ Dirección de Impulso a la Innovación y Desarrollo \\ Carretera Ensenada-Tijuana 3918 Zona Playitas C. P. 22860 Ensenada, Baja California, México
}

\begin{abstract}
Videoconferencing transmission over wireless channels presents relevant challenges in mobile scenarios at vehicular speeds. Previous contributions are focused on the optimization of the transmission of multimedia and delay-sensitive applications over the forward link. In this paper, a new Quality of Service (QoS) parameter adaptation scheme is proposed. This scheme applies the Cross-Layer Design technique on the reverse link of an 1xEV-DO Revision 0 channel. As the wireless channel parameters and the vehicle speed have significant influence in the network layer packet loss rate, it is proposed that the data rate generated by the application adapts itself to the throughput offered by the lower layers as a function of such packet loss rate. Simulations of the proposed model show a significant reduction in losses caused by wireless channel impairments and vehicle mobility, resulting in an improvement in the performance of the mobile videoconferencing session.
\end{abstract}

Keywords: Cross-Layer Design, EV-DO, Mobile videoconferencing, QoS, Reverse Link

\section{RESUMEN}

La transmisión de videoconferencia sobre canales inalámbricos presenta desafíos relevantes en escenarios móviles a velocidades vehiculares. Las contribuciones previas están enfocadas a optimizar la transmisión de aplicaciones multimedia y sensibles al retardo sobre el canal descendente. En este artículo se propone un nuevo esquema de adaptación de parámetros de Calidad de Servicio. Este esquema aplica la técnica del Diseño Entre Capas en el enlace ascendente de un canal 1xEV-DO Revisión 0 . Como los parámetros del canal inalámbrico y la velocidad del vehículo tienen influencia significativa en la tasa de pérdida de paquetes de capa de red, se propone que la tasa de datos generada por la aplicación se adapte al caudal eficaz ofrecido por las capas inferiores en función de dicha tasa de pérdida de paquetes. Simulaciones del modelo propuesto, muestran una reducción significativa en las pérdidas ocasionadas por las características del canal inalámbrico y la movilidad del vehículo, dando como resultado una mejora en el desempeño de la sesión de videoconferencia móvil.

\section{Introduction}

Multimedia traffic transmission over the forward link of mobile and wireless communications has been widely studied in recent years [1-11] due to the search of mechanisms that allow setting different levels of Quality of Service (QoS) for different information flows carried by actual Internet Protocol (IP) based-service converged networks. It is possible to find developments that allow the transmission of Video-on-Demand (VoD) and there is research oriented to the optimization of isolated layers [7]. Although not explicitly described in the literature $[1-11,13]$, research efforts have been oriented to the optimization of multimedia transmissions over the forward links of wide area wireless technologies, due to limitations on the peak data rates that can be achieved in the reverse links. In some current third generation cellular networks (e. g., EV-DO Rev. A) deployed in countries like Mexico, the links are still asymmetric [13]. This condition limits the use of multimedia 
applications like videoconferencing, which require similar capabilities on both, forward and reverse links. Applications that support sending and receiving real-time audio and video flows, do not take advantage of the use of different QoS levels nor of the network channel state information. This is particularly significant when these applications are transmitted over wireless links, where the proneto-errors nature of the channel, causes delays and increases jitter and packet loss rate. In order to cope with such challenging environment, new techniques like Cross-Layer Design (CLD) have been object of recent research [6-7, 9-11]. The aim of using CLD is to improve the whole communications process, emphasizing the optimization and the exchange of state parameters between not adjacent layers; in an opposite way to the traditional Open Systems Interconnection (OSI) inter-layer communications.

In this work, a QoS parameter adaptation scheme based on CLD is proposed and evaluated through simulations. The proposal is oriented to optimize the packet loss rate experienced in the transmission of video from a mobile terminal (reverse link) traveling at $80 \mathrm{~km} / \mathrm{h}$, within a cdma2000 1xEV-DO Revision 0 cell network coverage.

The main point of this paper is the dynamic adaptation of QoS parameters by means of a crosslayer parameter abstraction process of an H.263 video encoder. Receiving the appropriate indications from a cross-layer optimizer, the video encoder can update the frames per second (fps) and the video resolution format as a function of the throughput that is being offered by the lower layers. The use of an 1xEV-DO Revision 0 empirical channel model [12] allows us to consider the offered throughput from the network layer level, because such channel model offers a relationship between the Packet Loss Rate (PLR) at the network layer and an indirect estimation of the reverse link channel state.
The remainder of this paper is organized as follows: In Section 2 we present the modular crosslayer architecture used for this work and we detail the processes involved in the whole cross-layer optimization. The results of our simulations are presented in Section 3. Finally, in Section 4 we present the conclusions of the paper.

\section{Cross-layer architecture}

In this work we follow the cross-layer architecture proposed in [7]. Such architecture consists of the processes of parameter abstraction, cross-layer optimization, and decision distribution. The considered simulation scenario is showed in Figure 1. In this scenario, the mobile unit has already established both, an 1xEV-DO Revision 0 (also referred as DO Rev 0) [13] link and, a mobile videoconference session. Our efforts are focused on the reverse link due to its limitations, mainly in regard to the peak data rate. The aim of the proposed Cross-Layer design is to dynamically modify the fps and format resolution (with which the real-time sequence video is encoded) as the channel's state varies, resulting in a modification of the data rate generated by the application, changing in consequence, the throughput demanded to lower layers. It is well known that if the radio link layers (PHY and MAC layers) are not able to satisfy the demand of throughput from upper layers, a queuing condition is generated. Normally, this occurs due to bad channel conditions, network congestion or because the amount of data sent to lower layers is simply greater than the peak rate offered by the communication technology. This queuing condition introduces delay and reduces the actual throughput perceived by the upper layers. If the channel conditions are varying, the size of the queues will also vary, generating more jitter. Then, if the data rate is dynamically adapted to offered throughput, queue's size is kept at minimal levels, reducing delay, jitter and decreasing losses in the communication process. 
The system architecture is illustrated in Figure 2. It can be observed that a long-term bottom-up approach is considered since the optimization is carried out taking into account radio layer specific parameters and adapting application layer specific parameters to them.
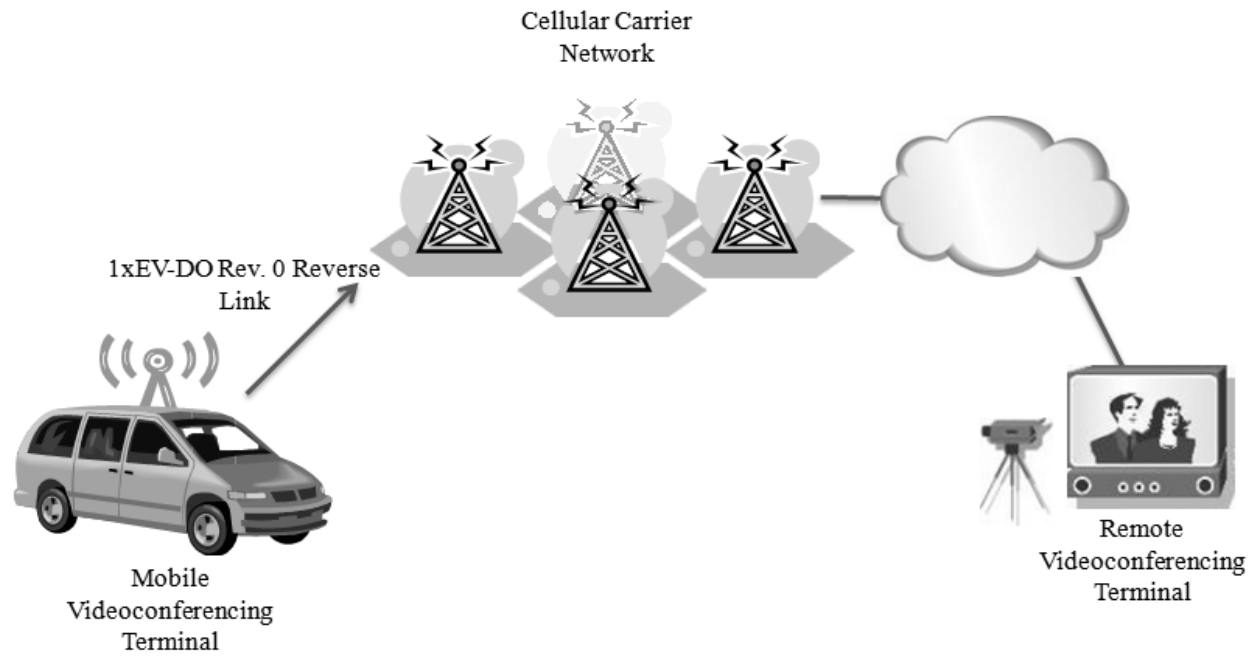

Figure 1. Considered simulation scenario. The proposal is oriented to optimize the data losses experimented in the transmission of video from a mobile terminal (reverse link) over a cdma2000 1xEV-DO Revision 0 link at vehicular speeds
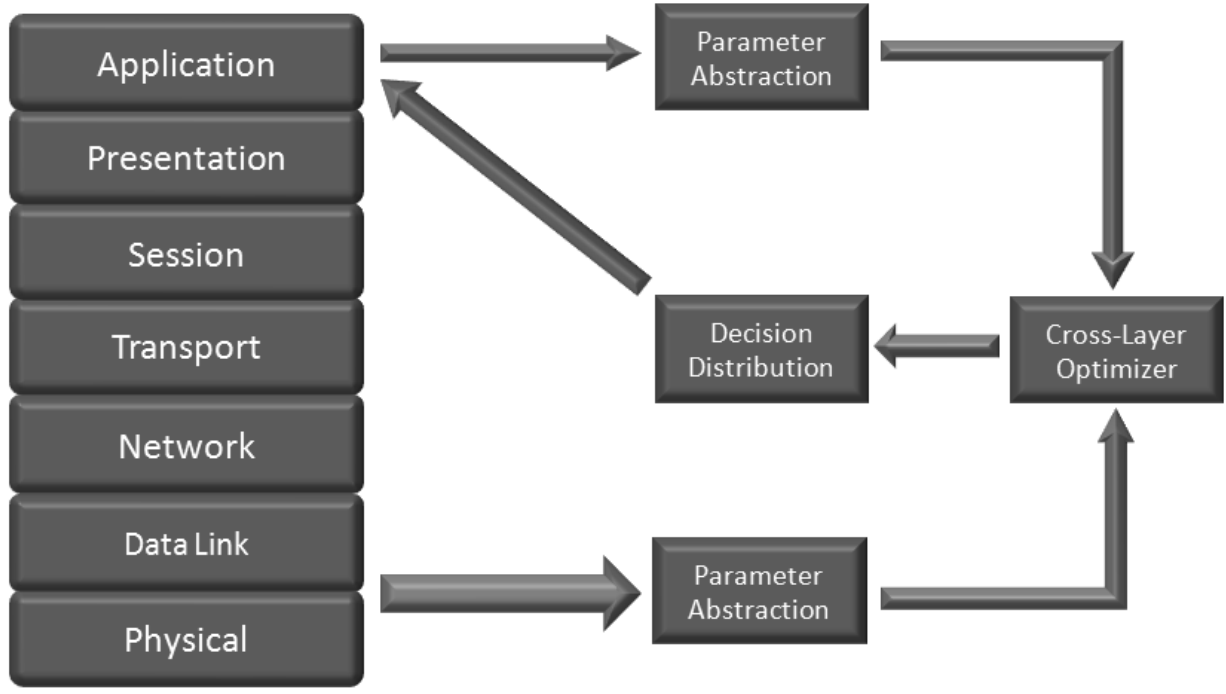

Figure 2. Cross-layer architecture system: parameter abstraction, cross-layer Optimizer and decision distribution 
However, in order to achieve such optimization, application layer-specific parameters are abstracted into a more useful parameter at network layer level (data rate). Radio layer specific parameters are abstracted into a network layer PLR. In this way, optimization is completed at an intermediate level.

\section{1 Parameter abstraction}

The parameter abstraction is a required process in CLD because parameters of a specific layer may not be adequately interpreted in other layers. All participating layers in the optimization process require its own parameters abstraction process. As we have two layers (Radio Link and Application) involved in the optimization process, we describe the abstraction process for each one of them as follows.

\section{1.1 Radio link layer parameter abstraction}

We define the set of radio layer specific parameters as $R=\left\{r_{1}, r_{2}, \ldots\right\}$ of tuples $r_{i}=\left(P_{i}, \mathrm{f}_{d_{i}}\right)$ [7]. Where $P$ is the instant Received Signal Strength Indicator (RSSI) value of the DO Rev 0 access terminal (mobile host), read as an indirect estimation of the reverse link channel state and, $f_{d}$ is the maximum doppler shift. As these parameters can take multiple values, the set $R$ contains all possible combinations of them and each tuple $r_{i}$ represents one possible combination. In order to formalize the parameter abstraction, we define the abstracted parameters set $\tilde{R}=\left\{\tilde{r}_{1}, \tilde{r}_{2}, \ldots\right\}$ of tuples $\tilde{r}_{i}=\left(P L R_{i}\right)$, where $P L R_{i}$ is the $i$-th packet loss rate at network layer that would be perceived at the $r_{i}$ combination of RSSI value and maximum doppler shift. The relationship between the set $R$ of all possible tuples of radio layer parameters and the set $\tilde{R}$ of all possible tuples of abstracted radio layer parameters is defined by the relation $\mathrm{B} \subseteq R \times \tilde{R}$ with domain in $R$ and codomain in $\tilde{R}$ and where operator $\times$ denotes the Cartesian product. Relation $B$ is defined in such way that contains only all valid pairs $\left(r_{i}, \tilde{r}_{i}\right)$. Set $\mathrm{B}$ is then called the radio link layer parameter abstraction.

This formalization of radio layer parameter abstraction process is not technology specific, and in contrast to the abstraction process performed in [7], our process was accomplished in an experimental way through the use of an empirical channel model that is specific for DO Rev 0 reverse link when the mobile is traveling at $80 \mathrm{~km} / \mathrm{h}(22.22$ $\mathrm{m} / \mathrm{s}$ approximately). The small circles shown in Figure 3 were obtained through more than 125 field measurements. In the experiments, the power values indicated by the RSSI of a mobile EVDO Rev. 0 terminal and the PLR experienced in the transmission were measured. The experimental arrangement assures that the measured PLR is affected only by the variations of the reverse link [12]. The value of the RSSI was considered as an indirect estimator of the reverse link's channel state due to the impossibility of making measurements in the base stations of the services provider. The results show that a high correlation exists between the values of the RSSI and the levels of the PLR, which proofs that the estimation is adequate.

In the experiments, a personal computer established, maintained and ended a videoconferencing session. In the start of every experiment, the vehicle was already traveling at 80 $\mathrm{km} / \mathrm{h}$ and the speed was kept constant during the videoconferencing session. In order to reproduce the same conditions on every test, a 10 seconds video sequence was previously recorded and it was transmitted on every experiment as if it would be a live videoconferencing session. The duration of the video sequence was chosen as a function of the minimal fps rate achieved in the tests ( $6 \mathrm{fps}$ ) and common values of the group of picture (GOP) [7, $15]$, in such a way that at least one GOP is transmitted. 


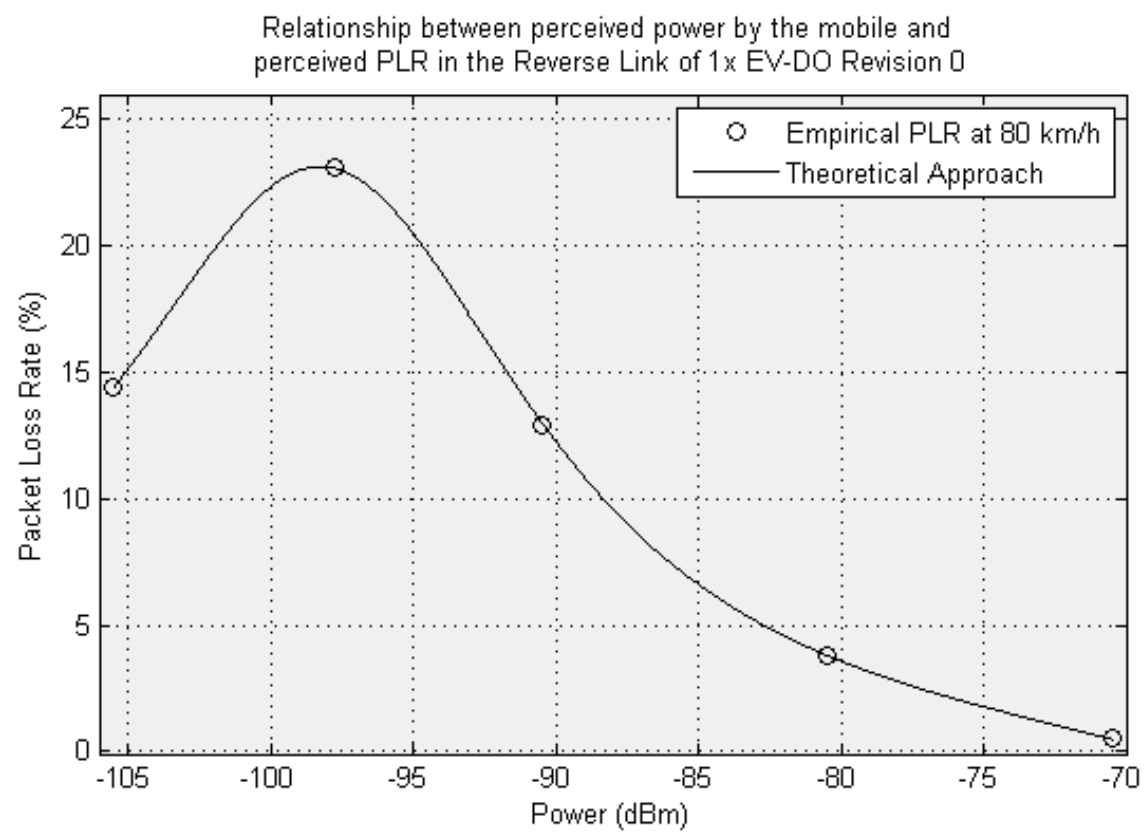

Figure 3. Relationship between power perceived by the mobile terminal and experienced PLR in the Reverse Link of 1x EV-DO Revision 0

The PLR values obtained on each experiment were averaged and they are represented as empirical values on Figure 3 . With the objective of estimating the PLR for values of power different from those measured (Radio layer parameter abstraction), a theoretical approach of the empirical values was carried out using the nonlinear least squared method with the Levenberg-Marquardt algorithm for adjustment of coefficients. The theoretical approach is described as

$$
f(x)=\frac{2457 x+169580}{x^{3}+257.3 x^{2}+21709 x+594487}
$$

Where $f(x)$ is the estimated PLR and $x \in[-105.5,-70.5]$ is the actual value of power, measured in $\mathrm{dBm}$, that the DO Rev 0 card used in the experiments can detect [12]. The results obtained with this semi-empirical model for the reverse link of EV-DO Rev. 0 are consistent to those previously obtained by [10].

\section{1.2 Application layer parameter abstraction}

The application layer parameter abstraction process is described in a similar way. Let $A$ the set of application layer specific parameters defined by

$$
A=\left\{a_{1}, a_{2}, \ldots\right\} \text { with tuples } a_{i}=\left(\mathrm{f}_{\text {res }_{i}}, \mathrm{fps}_{i}\right) \text {, }
$$

where $f_{\text {res }}$ is the format video resolution and fps is the frames per second rate used by the video encoder. As these parameters can take several values, set $A$ contains all possible combinations and each tuple $a_{i}$ represents one of them. We also define the set $\tilde{A}=\left\{\tilde{a}_{1}, \tilde{a}_{2}, \ldots\right\}$ of tuples $\tilde{a}_{i}=\left(d_{i}\right)$ of abstracted parameters, where $d_{i}$ is the $\mathrm{i}$-th data rate that the video application can generate. The relationship between set $A$ of all possible tuples of application layer parameters and set $\tilde{A}$ of all possible tuples of abstracted application layer parameters is defined by the relation $\Delta \subseteq A \times \tilde{A}$ with domain in $A$ and codomain in $\tilde{A}$ and where $\times$ denotes the 
Cartesian product. It is established that relation $\Delta$ contains only all valid pairs and that $\Delta$ is a total function. We define relation $\Delta$ as a total function due to the fact that in the decision distribution process, parameter abstraction is reverted and some value $d_{i}$ could correspond to more than one video resolution format and fps combination. Under these circumstances, it would exist an ambiguity generating undesirable instabilities in the whole process as stated in [14].

The abstraction process described here considers that a change in the video resolution format or in the fps rate, modifies the traffic generated by the application, such behavior is also a function of the video encoder. These specific application layer parameters have an important role in the amount and shape of traffic that is received by lower layers for transmission over the physical media. In [12] is possible to find a characterization of the generated traffic at network layer level by an $\mathrm{H} .263$ video encoder when the video resolution format and fps rate are modified; in our simulations such characterization was used as our application layer parameter abstraction. The characterization shows that when the encoder is sending I-frames, the peak data rate is achieved. Subsequent P-frames generate lower data rates, however, depending on the amount of movement in the video sequence, Iframes can be generated often. As described in [7], loosing I-frames has dramatic influence in the distortion profile. In this way, it is convenient to assume a constant bit rate (CBR) traffic source for an H.263 video encoder, setting the bit rate to the peak. This assumption, although does not describe the real behavior of the traffic generated by the application, will assure that the cross-layer design is going to execute decisions considering the most critical video information. Figure 4 shows the performed traffic characterization of an H.263 encoder.

Data rate generated for different video parameters using $\mathrm{H} .263$

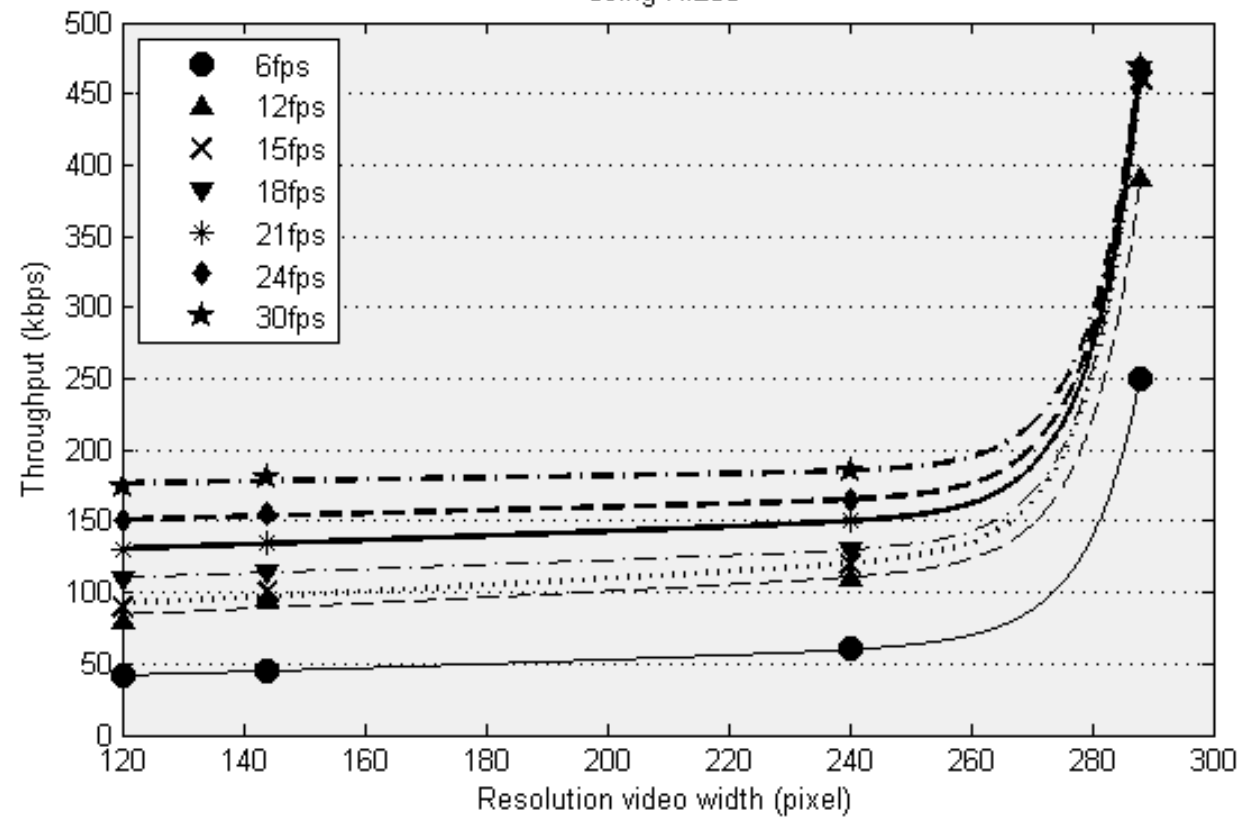

Figure 4. Data rate generated for different video parameters using H.263 


\subsection{Cross-layer optimization}

In order to start the cross-layer optimization, we redefine the set $\tilde{A}$ as

$$
\tilde{A}=\left\{\tilde{a}_{1}, \tilde{a}_{2}, \ldots, \tilde{a}_{i-2}, \tilde{a}_{i-1}, \tilde{a}_{i}\right\}
$$

with $\tilde{a}_{i}<\tilde{a}_{i+1}$ for all $i$. Abstracted parameters sets $\tilde{A}$ and $\tilde{R}$ from both, the application layer and the radio link layer, are the inputs to the cross-layer optimizer. Since any combination of abstracted parameters tuples is valid, we define the crosslayer parameter set $\tilde{X}=\tilde{R} \times \tilde{A}$ of tuples $\tilde{x}_{m}=\left(\tilde{r}_{i}, \tilde{a}_{i}\right)$. We define the operation of the cross-layer optimizer $\Omega$ as

$$
\Omega: \tilde{X} \rightarrow \tilde{Y} \subset \tilde{X}
$$

where $\tilde{Y}$ is a not empty set and it is assumed that $|\tilde{Y}|=1$ The only element present in $\tilde{Y}$ is such that $\tilde{Y}=\left\{\tilde{X}_{\text {opt }}\right\} \in \tilde{X}$. The cross-layer optimizer output $\tilde{x}_{\text {opt }}$ is defined with respect to a particular objective function as

$$
\mathrm{O}: \tilde{X} \rightarrow \mathfrak{R}
$$

Where $\mathfrak{R}$ is the set of real numbers. As different tuples $\tilde{X} \in \tilde{X}$ can be optimal for different goals of design, we propose that omicron function be defined by the correspondence rule:

$$
\mathrm{O}(\tilde{x})=\left\{\begin{array}{llr}
\left(\tilde{a}_{i+1}\right) & \text { if } & \tilde{a}_{i}\left(1-\tilde{r}_{\max }\right) \leq \tilde{a}_{i}\left(1-\tilde{r}_{i}\right)<\tilde{a}_{i}\left(1-\frac{2}{3} \tilde{r}_{\max }\right) \\
\left(\tilde{a}_{i}\right) & \text { if } & \tilde{a}_{i}\left(1-\frac{2}{3} \tilde{r}_{\max }\right) \leq \tilde{a}_{i}\left(1-\tilde{r}_{i}\right)<\tilde{a}_{i}\left(1-\frac{1}{3} \tilde{r}_{\max }\right) \\
\left(\tilde{a}_{i-n}\right) & \text { if } & \tilde{a}_{i}\left(1-\frac{1}{3} \tilde{r}_{\max }\right) \leq \tilde{a}_{i}\left(1-\tilde{r}_{i}\right)<\tilde{a}_{i}\left(1-\tilde{r}_{\min }\right)
\end{array}\right.
$$

Where $\tilde{a}_{i}$ is the actual data rate generated by the application (application layer abstracted parameter), $\quad \tilde{r}_{\max }$ and $\tilde{r}_{\min }$ are respectively, the maximum and the minimum PLR at network layer level that can be achieved in our channel model (radio link layer abstracted parameter), $\tilde{r}_{i}$ is the actual PLR at network layer level and, the tuple $\tilde{a}_{i-n}$ guarantees that:

$$
\tilde{a}_{i}\left(1-\tilde{r}_{i}\right)-\tilde{a}_{i-n}>0
$$

With $n \in[1, i]$. The tuple $\tilde{a}_{i-n}$ allows the crosslayer optimizer to react quickly to abrupt changes in PLR because it adjusts the data rate generated by the video application toward the closest value of the throughput offered by the radio link layers. Note that the optimizer should first determine which $n$ satisfies the condition (6) prior to the search of the tuple $\tilde{x}_{\text {opt }}$. Also note that the output of the omicron function only makes reference to the application layer abstracted parameters from the tuples $\tilde{x}_{n}=\left(\tilde{r}_{i}, \tilde{a}_{i}\right)$, since the tuples $\tilde{r}_{i}$ are not modifiable in any way. Tuples $\tilde{r}_{i}$ are used by the omicron function to determine the throughput at network layer level offered by radio link layers. The cross-layer optimizer considers a quasi-stationary channel from the moment it receives a tuple $\tilde{x}_{m_{1}}$ and until it receives a new tuple $\tilde{x}_{m_{2}}$. In order to have accurate information of the radio channel state, the cross-layer optimizer must request information periodically to the radio link layers. How often such information should be prompted is related to the so-called, decorrelation time $T_{d e c}$, as it will be described in Section 3.

The design goal for which omicron function searches the optimal value $\tilde{x}_{\text {opt }}$ is the reduction of data losses due to differences between the actual throughput offered by the radio link layers, and 
the data rate with which the application generates data. The function defined by (5), finds the tuple $\tilde{a}_{i}$ that is the closest to the actual throughput $\tilde{a}_{i}\left(1-\tilde{r}_{i}\right)$ offered by the lower layers. Note that the offered throughput is always lower than the actual data rate since according to (1), $\tilde{r}_{i}$ is never zero. Offered throughput can take as many values per data rate $\tilde{a}_{i}$ as power levels can be both, detected and signaled, by the DO Rev 0 mobile terminal. However, the set $\tilde{A}$ has a discrete and finite number of elements whose values are also discrete, and they are a function of the video coding parameters. In this way, the optimal value can be found by the evaluation of the difference between the data rate and the offered throughput. The function defined by (5) considers three decision-zones in which the offered throughput could be found in relation to the actual data rate that the application generates. Such decisionzones are defined considering the maximum and the minimum PLR according to (1) and in this case, all zones have the same size.

\subsection{Decision distribution}

Following the formalism introduced in previous sections, the output of the cross-layer optimizer $\tilde{x}_{\text {opt }}=\left(\tilde{r}_{\text {opt }}, \tilde{a}_{\text {opt }}\right)$ should be signaled by the decision distributor to appropriate layers. In our study, the cross-layer optimizer signals the indicated data rate $\tilde{a}_{i}$ that the application should generate in order to minimize losses due to variations in the radio channel. The element $\tilde{r}_{\text {opt }}$ is not signaled by (5) due to the impossibility of making any modification to the channel state, in other words $\tilde{r}_{\text {opt }}=\tilde{r}_{i}$. However, $\tilde{a}_{\text {opt }} \in \tilde{A}$ must be notified to the application layer and the parameter abstraction process should be then, reverted as:

$$
\tilde{a}_{o p t} \in\left\{a \mid\left(a, \tilde{a}_{o p t}\right) \in \Delta\right\}
$$

Given that $\Delta$ is a total function, the value of $a$ that maps to $\tilde{a}_{\text {opt }}$ is unique.

\section{Simulation results}

Performed simulations consider a mobile terminal transmitting 10 seconds of an H.263 coded video sequence over a DO Rev 0 reverse link. The videocoding parameters are chosen considering the maximum peak data rate that can be achieved in the reverse link of DO Rev 0 (153.6 kbps) [13] and, at the beginning of every simulation, the video encoder is generating the maximum data rate. In this scenario we assume that the mobile terminal has a constant speed of $80 \mathrm{~km} / \mathrm{h}$ and these conditions are such that the perceived power goes from $-105.5 \mathrm{dBm}$ to $-70.5 \mathrm{dBm}$. It is considered that the mobile terminal can read any value of power in this range. Size packet at network layer level is considered constant.

As the proposed cross-layer design is going to adapt the traffic generated by the application to the throughput offered by radio link layers, there are two parameters that should be considered: radio channel state sampling time $T_{s}$, and application response time $T_{\text {ans. }} T_{s}$ is the period of time used by the CLD to sample and obtain knowledge of the channel state; $T_{\text {ans }}$ is the period of time taken by the application to modify video parameters in response to a cross-layer signaling. In simulations, it is also common to assume that the channel's quality is constant in decorrelation time $T_{\text {dec }}$. This time is a function of the wavelength, mobile speed, and mobile surrounding conditions [4]. After time $T_{d e c}$, the channel quality takes a new random value. Given this, the Cross-Layer optimizer should read the channel's quality at least in $T_{d e c}$ in order to have accurate information.

Figure 5 shows the data rate generated by the application and the radio link layer offered throughput when $T_{\text {ans }}=200 \mathrm{~ms}$ and 


\section{Cross-Layer optimization of the packet loss rate in mobile videoconferencing applications, R. Rivera-Rodríguez et al., 130-143}

$T_{s}=T_{\text {dec }}=2 \mathrm{~ms}$. Throughput offered by lower layers is obtained from

$\mathrm{Th}_{\mathrm{of}}=\left\{\begin{array}{lll}d(1-P L R) & \text { if } & d \leq d_{\max } \\ d_{\max }(1-P L R) & \text { if } & d>d_{\text {max }}\end{array}\right.$

where $d$ is the data rate generated by the application, $d_{\max }$ is the maximum data rate offered by the radio technology (uplink of EV DO Rev 0 in this case), and PLR is the packet loss rate perceived at the network layer level.

Losses in Figure 5 are obtained from the difference between $d$ and $\mathrm{Th}_{\text {of }}$. Note that $\mathrm{y}$-axis is labeled
"Equivalent Throughput"; this is due to the consideration that every dot in Figure 5 represents a throughput value that would be generated if the transmission would last, at least, one second. Therefore it is adequate to use kbps as units in this and subsequent figures.

Figure 6 shows graphically the significant reduction in losses due to the adaptation of the generated data rate. The curve representing data losses when CLD is implemented has such shape due to dynamic adaptation of the data rate generated by the application in response to cross-layer optimizer notifications.

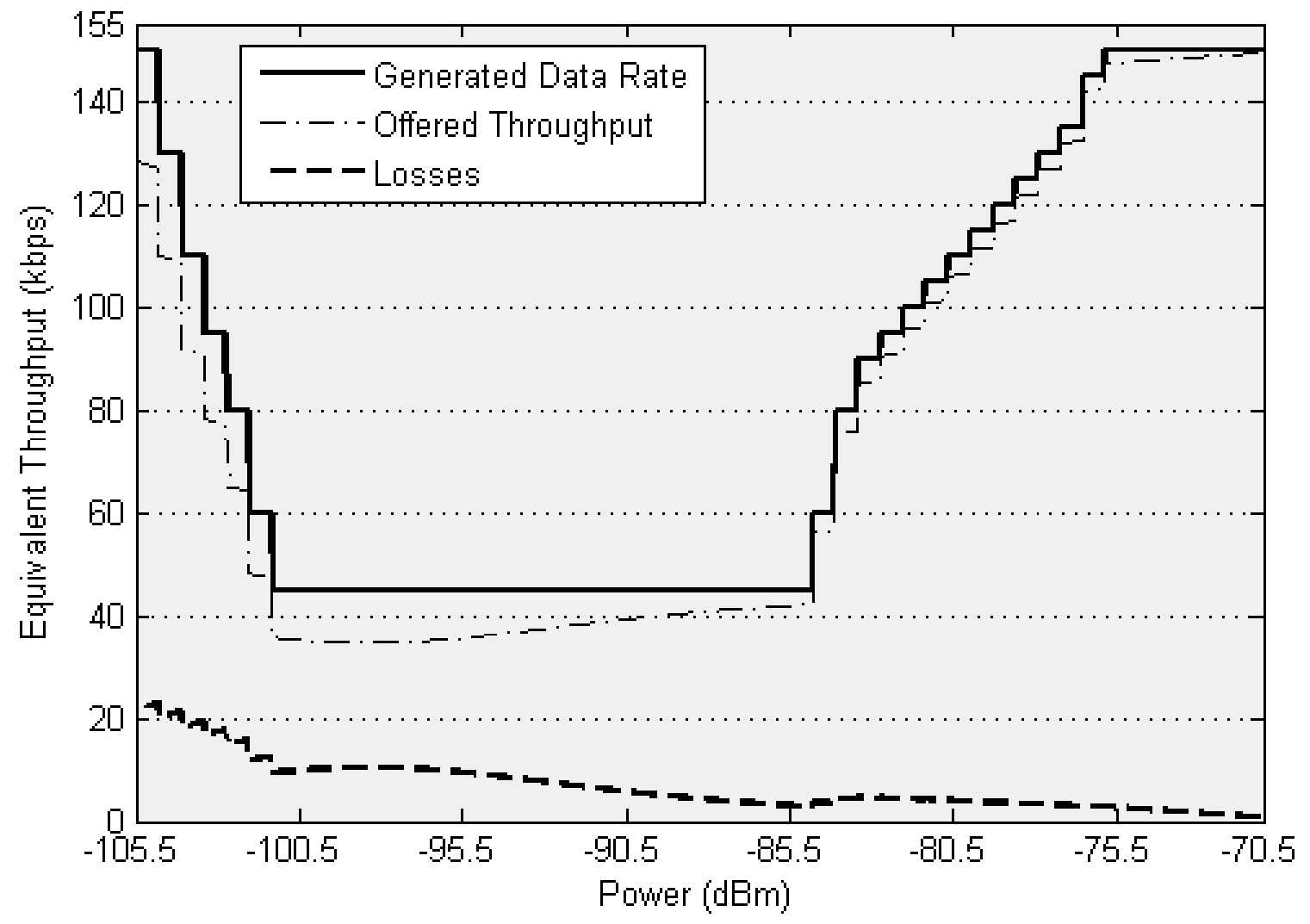

Figure 5. Data rate generated by the application and the radio link layer offered throughput when $T_{\text {ans }}=200 \mathrm{~ms}$ and $T_{s}=2 \mathrm{~ms}$ 


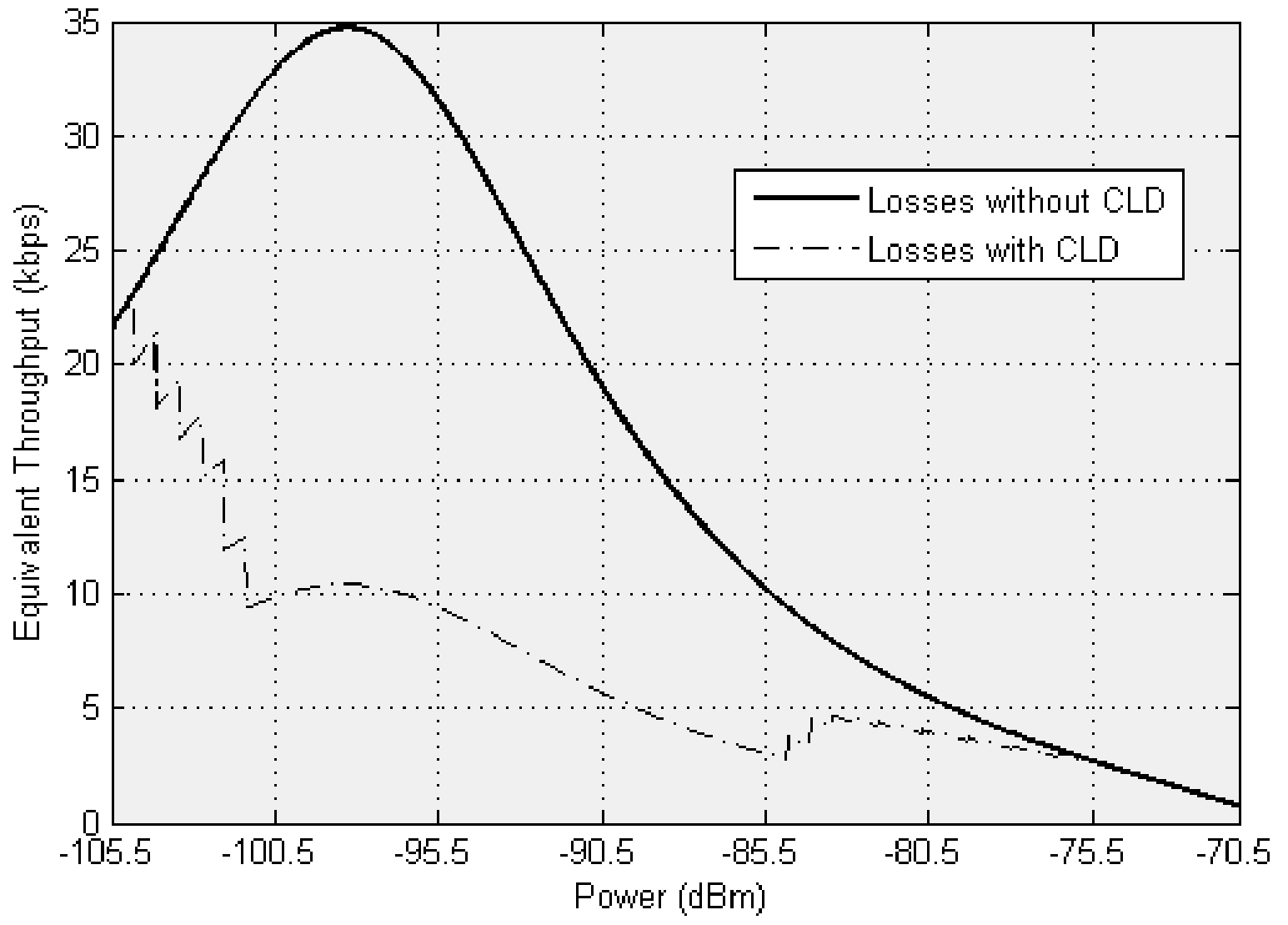

Figure 6. Reduction in losses due to the generated data rate adaptation to the offered throughput when $T_{\text {ans }}=200 \mathrm{~ms}$ and $T_{s}=2 \mathrm{~ms}$

Figures 7 and 8 show simulations results when $T_{\text {ans }}=1000 \mathrm{~ms}$ and $T_{s}=100 \mathrm{~ms}$. It is possible to note that the behavior of the data rate is more stable and that during the video sequence test, the minimal data rate is not achieved because the application takes more time to respond to the cross-layer notifications. This condition also generates a larger amount of losses than in the previous case. It can be observed that although channel sampling time $T_{s}$ is 50 times the decorrelation time $T_{\text {dec }}$, the behavior of the cross-layer optimizer is not apparently compromised. A detailed view of Figure 7 would show that, what we have called virtual offered throughput, is indeed different from the real offered throughput. The former is the offered throughput that the cross-layer optimizer sees because of the bigger sampling time $T_{s}$, the latter is the real throughput that is being offered by the radio link layers. 


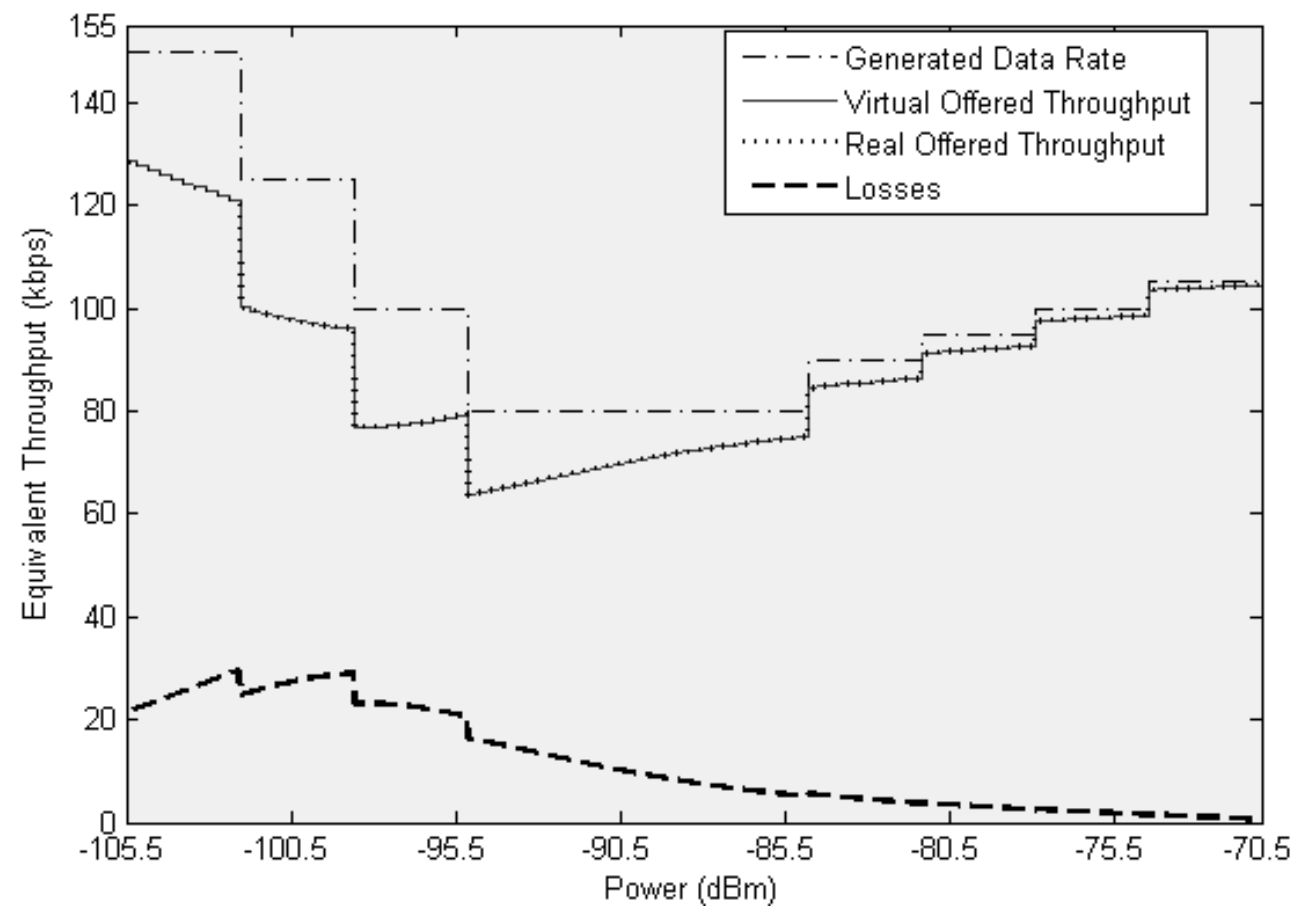

Figure 7. Data rate generated by the application and the radio link layer offered throughput when $T_{\text {ans }}=1000 \mathrm{~ms}$ and $T_{s}=100 \mathrm{~ms}$

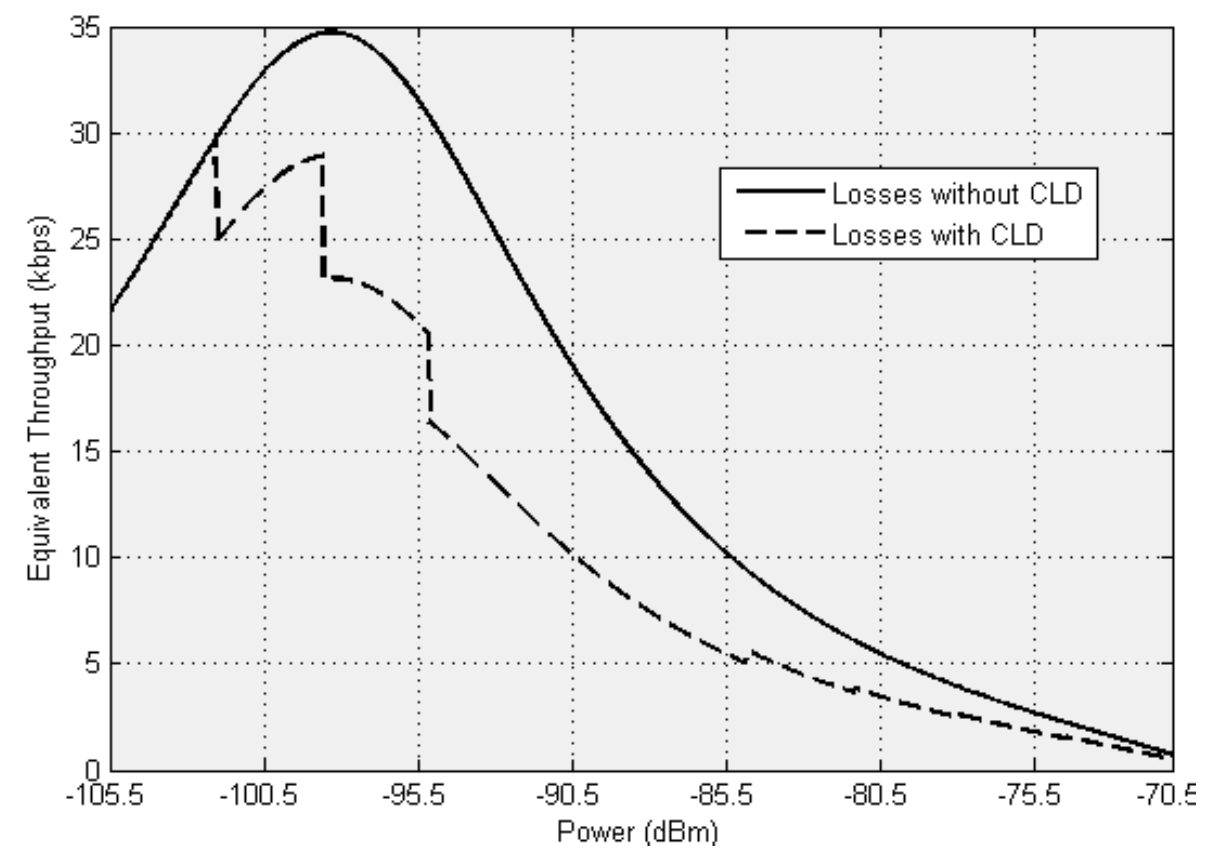

Figure 8. Reduction in losses due to the generated data rate adaptation to the offered throughput when $T_{\text {ans }}=1000 \mathrm{~ms}$ and $T_{s}=100 \mathrm{~ms}$ 
Our results show that a qualitative improvement was reached when the proposed cross-layer design was implemented. An extensive quantitative analysis developed in [12] shows that a Cross-Layer design as the one presented in this article can be conducive to reductions in the order of $50 \%$ of the data losses when the data rate generated by the application is adapted to the offered throughput by the radio link layer.

\section{Conclusions}

A new QoS parameter adaptation scheme based on cross-layer design is proposed. This scheme was designed to reduce losses in video data transmission over a 1xEV-DO Revision 0 reverse link when the mobile terminal is traveling at vehicular speeds. The cross-layer design presented in this paper is highly adaptive and scalable as the one proposed in [7]. Our approach can be adapted easily to other radio technologies or to other video encoders with the simple exchange of a parameter abstraction module. In this work we have made use of both, empirical and semi-empirical approaches in the cross-layer parameter abstraction processes. Our simulations show that significant loss reduction can be achieved in the transmission of video sequences by using the design described in this paper. Although the best results are obtained when the time response of the application layer tends to be small, it should be considered that video encoders take a few seconds to react to cross-layer notifications and this implies more losses. In this way, further research regarding the distribution decision process is required in order to determine the impact that the proposed CLD has on the quality of the video sequence when changes in video format resolution or $\mathrm{fps}$ rate are signaled. There is a tradeoff between the stability in the behavior of the application and the reduction of losses. The granularity of the layers involved in the cross-layer process must be considered if it is desired to keep as close as possible the channel state sampling time to the decorrelation time.

\section{References}

[1] Shan, Y., \& Zakhor, A. Cross Layer Techniques for Adaptive Video Streaming Over Wireless Networks. Proceedings of the International Conference on Multimedia (ICME '02), 2002, vol. 1, pp. 277-280, Lausanne, Switzerland, August.

[2] Stockhammer, T., Hannuksela, M. M., \& Wiegand, T. H.264/AVC in Wireless Environments. IEEE transactions On Circuits and Systems For Video Technology, Vol. 13 No. 7, July 2002, pp. 657-673.

[3] Choi, L., Kellerer, W., \& Steinbach, E. Cross Layer Optimization for Wireless Multi-User Video Streaming. Proceedings of the International Conference on Image Processing (ICIP '04), 2004, pp. 2047-2050. Singapore, Republic of Singapore, October.

[4] Choi, L., Ivrlac, M., Steinbach, E., \& Nossek, J. Analysis of Distortion Due to Packet Loss in Streaming Video Transmission over Wireless Communications Links. Proceedings of the International Conference on Imaging Processing (ICIP'05), 2005, pp. 189-192. Genoa, Italy, September.

[5] Ksentini, A., Naimi, M., \& Guéroui, A. (2006). Toward an improvement of H.264 Video Transmission over IEEE 802.11e through a Cross-Layer Architecture. IEEE Communications Magazine, Vol. 44 No. 1, January 2006, pp. 107-114.

[6] Khan, S., Steinbach, E., Sgroi, M., \& Kellerer, W. Application-Driven Cross-Layer Optimization for Video Streaming over Wireless Networks. IEEE Communications Magazine, Vol. 44 No. 1, January 2006, pp. 122-130.

[7] Choi, L.-U., Kellerer, W., \& Steinbach, E. On CrossLayer Design for Streaming Video Delivery in Multiuser Wireless Environments. EURASIP Journal on Wireless Communications and Networking, Vol. 2006, 2006 , pp. 1-10. 


\section{Cross-Layer optimization of the packet loss rate in mobile videoconferencing applications, R. Rivera-Rodriguez et al., 130-143}

[8] Yavuz, M., Diaz, S., Kapoor, R., Grob, M., Black, P., Tokgoz, Y., et al. VolP over cdma2000 1xEV-DO Revision A, IEEE Communications Magazine, Vol. 44 No. 2, February 2006, pp. 88-95.

[9] Triantafyllopoulou, D., Passas, N., Lampropoulos G., Kaloxylos, A. Joint Application and Physical Layer Adaptation for improved Performance in Wireless Networks, Proc. IEEE International Symposium on Wireless and Pervasive Computing (ISWPC), 2008, pp. 256-260, Santorini, Greece, May.

[10] Yoo, S., Jung, S.M., Kim, B.S., Yun, H.Y., Kim, S.R., Kim, D.K. Prototype Design of Mobile Emergency Telemedicine System in LNCS 3481, Springer Berlin / Heidelberg, 2005, pp. 1028-1034.

[11] Juan, H., Huang, H., Huang, C., Chiang, T. Crosslayer System Designs for Scalable Video Streaming over Mobile WiMAX. Proceedings of the Wireless Communications and Networking Conference, 2007, pp. 1860-1864, Hong Kong, March.

[12] Olivares O., Automatic QoS parameters adjust in mobile videoconferencing using cross layer design for $3 \mathrm{~g}$ EVDO wireless networks, Dept. of Elec. and Telecomm., CICESE. Ensenada, Baja California. M. Sc. Dissertation. 2008.

[13] Bhushan, N., Loth, C., Black, P., Attar, R., Jou, Y.-C., Fan, M., et al. CDMA2000 1xEV-DO Revision A: A Physical Layer and MAC Layer Overview. IEEE Communications Magazine, Vol. 44 No. 2, February 2006, pp. 75-87.

[14] Kawadia, V., \& Kumar, P. A Cautionary Perspective on Cross-Layer Design. IEEE

Wireless Communications, Vol. 12 No. 1, 2005, pp. 3-11.

[15] Goshi, J., Ladner, R., Riskin, E., Mohr, A., Lippman, A. Unequal Loss Protection for H.263 Compressed Video, Data Compression Conference (DCC '03), 2003, pp. 73-82, Snowbird, USA, March. 


\section{Authors' Biography}

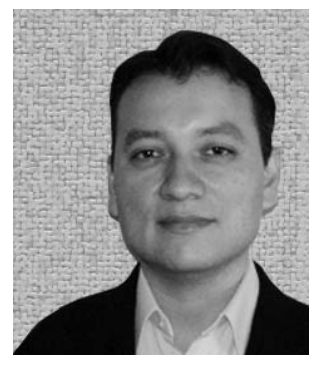

\section{Raul RIVERA-RODRIGUEZ}

He got his electronics engineering degree at the Sonora Institute of Technology (Mexico) in 1994. He got his M. Sc. degree in electronics and telecommunication at the Center for Scientific Research and Higher Education of Ensenada (CICESE, México) in 1997, during this period he had a research stay at the Départament Réseaux in the École Nationale Supérieure des Télécommunications (L'école Télécom ParisTech). He is member of the Wireless Communication Research Group in the Applied Physics Division at CICESE Research Center. His current research interests include the areas of network management systems, quality of service IP networks, signal processing for wireless communications, multimedia communications, communication networks, cross-layer design, and coding theory.

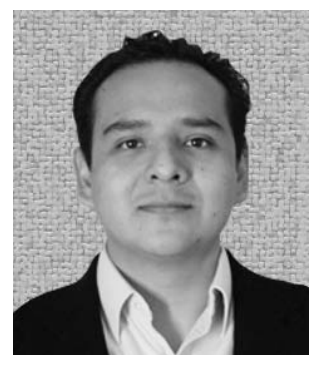

\section{Oscar Eduardo OLIVARES-DOMINGUEZ}

He got his telecommunications engineering degree at the Universidad del Valle de Mexico in 2004. He got the CCNA and CCAI certifications in 2005 and since then he has been IT instructor at different universities in Mexico and in the European University of Madrid, Spain. He got his M. Sc. degree in electronics and telecommunications at the Center for Scientific Research and Higher Education of Ensenada (CICESE, Mexico) in 2008. His current research interests are next generation IP networks, mobile multimedia communications, inter link satellite networks and cross-layer design.

\section{ArturO SERRANO-SANTOYO}

He earned his doctor's degree in electrical engineering from the National Polytechnic Institute in Mexico City in 1980. In 1981 he received the ALCATEL Annual Telecommunications Award for his contributions to rural satellite communications in Mexico, and in 1986 was honored with the ERICSSON Telecommunications Award. He has been a telecommunications consultant for the Organization of American States and the United Nations as well as for many private companies and governmental agencies. He was founder and executive director of Praxis Telecom and Teleddes Foundation. Dr. Serrano is member of the Mexican Academy of Engineering and author of the books "Telecommunications in Latino America" and "The Digital Divide: Myths and Realities". He is currently director of Innovation and Development at CICESE Research Center in Ensenada, Baja California, Mexico. 